\title{
Mars environment and magnetic orbiter model payload
}

\author{
B. Langlais - F. Leblanc - T. Fouchet - S. Barabash • \\ D. Breuer - E. Chassefière - A. Coates • \\ V. Dehant • F. Forget • H. Lammer · S. Lewis • \\ M. Lopez-Valverde · M. Mandea - M. Menvielle • \\ A. Pais · M. Paetzold • P. Read - C. Sotin • \\ P. Tarits - S. Vennerstrom - G. Branduardi-Raymont • \\ G. Cremonese • J. G. M. Merayo • T. Ott • \\ H. Rème · J. G. Trotignon · J. E. Walhund
}

Received: 30 October 2007 / Accepted: 6 May 2008 / Published online: 26 June 2008

(C) Springer Science + Business Media B.V. 2008

\author{
B. Langlais $(\varangle) \cdot$ C. Sotin \\ Laboratoire de Planétologie et de Géodynamique, \\ Université de Nantes, Nantes, France \\ e-mail: benoit.langlais@univ-nantes.fr \\ F. Leblanc $\cdot$ E. Chassefière \\ Service d'Aéronomie du CNRS/IPSL, \\ Université Pierre et Marie Curie, \\ Paris, France \\ T. Fouchet \\ Observatoire de Paris, \\ Paris, France \\ S. Barabash · J. E. Walhund \\ Swedish Institute of Space Physics, \\ Uppsala, Sweden \\ D. Breuer \\ DLR, Institute of Planetary Research, \\ Berlin, Germany
}

\section{A. Coates}

Department of Space and Climate Physics,

University College London, London, UK

V. Dehant

Royal Observatory of Belgium,

Uccle, Belgium

F. Forget

Laboratoire de Météorologie Dynamique/IPSL,

Paris, France 
Abstract Mars Environment and Magnetic Orbiter was proposed as an answer to the Cosmic Vision Call of Opportunity as a M-class mission. The MEMO mission is designed to study the strong interconnections between the planetary interior, atmosphere and solar conditions essential to understand planetary evolution, the appearance of life and its sustainability. MEMO provides a high-resolution, complete, mapping of the magnetic field (below an altitude of about $250 \mathrm{~km}$ ), with an yet unachieved full global coverage. This is combined with an in situ characterization of the high atmosphere and remote sensing of the middle and lower atmospheres, with an unmatched accuracy. These measurements are completed by an improved detection of the gravity field signatures associated with carbon dioxide cycle and to the tidal deformation. In addition the solar wind, solar EUV/UV and energetic particle fluxes are simultaneously and continuously monitored. The challenging scientific objectives of the MEMO mission proposal are fulfilled with the appropriate scientific instruments and orbit strategy. MEMO is composed of a main platform, placed on a elliptical $(130 \times 1,000 \mathrm{~km})$, non polar $\left(77^{\circ}\right.$ inclination $)$ orbit, and of an independent, higher apoapsis $(10,000 \mathrm{~km})$ and low periapsis $(300 \mathrm{~km})$ microsatellite. These orbital parameters are designed so that the scientific return of MEMO is maximized, in terms of measurement altitude, local time, season

H. Lammer

Space Research Institute, Austrian Academy of Sciences,

Vienna, Austria

\section{S. Lewis}

Department of Physics and Astronomy,

The Open University, Milton Keynes, UK

M. Lopez-Valverde

AIRE, Instituto di Astrofisica di Andalucia,

Granada, Spain

M. Mandea

GeoForschungsZentrum Potsdam,

Potsdam, Germany

M. Menvielle

CETP/IPSL,

Paris, France

A. Pais

Department of Physics, University of Coimbra,

Coimbra, Portugal

M. Paetzold

Universität zu Köln,

Cologne, Germany

P. Read

Atmospheric, Oceanic and Planetary Physics,

University of Oxford, Oxford, UK 
and geographical coverage. MEMO carry several suites of instruments, made of an 'exospheric-upper atmosphere' package, a 'magnetic field' package, and a 'low-middle atmosphere' package. Nominal mission duration is one Martian year.

Keywords Space vehicles • Instruments • Planets and satellites • General • Solar-terrestrial relations $\cdot$ Formation $\cdot$ Magnetic fields • Solar system • Formation

\section{Introduction}

Recent observations by the ESA spacecraft Mars Express, the two NASA Rovers Opportunity and Spirit [5], as well as by the NASA probe Mars Global Surveyor (MGS) and others, have changed our view on the evolution of Mars $[1,3,12]$. The most dramatic results concern the limited visible occurrence of minerals related to the action of water at its surface [24], the locally intense lithospheric magnetic fields [2], and the currently weak rate of ion escape driven by the solar wind [4]. These observations suggest that the period of a "wet Mars" was short-lived and that Mars lost much of its water by $3.5 \mathrm{Gyr}$ ago, when the magnetic field of Mars also died out [32].

P. Tarits

IUEM, Université de Brest,

Brest, France

S. Vennerstrom · J. G. M. Merayo

Danish National Space Center, Technical University of Denmark,

Copenhagen, Denmark

G. Branduardi-Raymont

Mullard Space Science Laboratory of University College London,

London, UK

G. Cremonese

Osservatorio Astronomico di Padova,

INAF, Padua, Italy

T. Ott

Swedish Space Corporation,

Solna, Sweden

H. Rème

CESR, Université de Toulouse,

Toulouse, France

J. G. Trotignon

Laboratoire de Physique et Chimie de l'Environnement,

CNRS, Orléans, France 
The geological structures still observed today suggest that Mars presented a global volcanic activity during the early Noachian (4.6-4.0 Gyrs) and a local volcanic activity during the late Noachian and Hesperian eras (4.03.2 Gyrs), while only a few isolated volcanic events took place during the Amazonian (3.2-present) following the crater-based Martian chronology [15]. Mars appears now as a cold, dry and possibly inactive planet: it has a very thin atmosphere, there is no liquid water at its surface, and there are no signs of current tectonic activity. The recently detected hydrated minerals (phyllosilicates) demonstrate that during the Noachian era, large aqueous reservoirs had existed at the surface (the Phyllosian era), before the environmental conditions turned more acidic (the Theiikian era) and eventually drier in the Amazonian and Hesperian eras corresponding to the Siderekian era following this alternate Martian chronology [6]. It is generally believed that the evolution of the Martian environmental conditions is linked with the extinction of the internal dynamo which would also have favoured atmospheric escape into space [10], emphasising the need for simultaneous and complementary observations of the current lithospheric magnetic field, the current atmospheric dynamics, and their interactions with the solar wind and plasma environment.

An integrated view of Martian matter and energy cycles, from the interior, through the surface and the atmosphere up to the solar wind interaction regions and beyond, is the essential next step toward deciphering the planet's evolution and past climate. The Mars Environment and Magnetic Orbiter mission is aimed at:

1. monitor temperature, wind and chemical composition throughout the seasonal cycle in order to characterize processes coupling low, middle and high atmospheric layers;

2. map the crustal magnetic field with an unprecedented spatial resolution that will allow a more precise timing of the evolution and cessation of the dynamo;

3. characterize the internal structure of Mars through electrical conductivity and gravity signatures;

4. elucidate the nature of the current systems formed by the interactions between the solar wind and the lithospheric field and investigate the dependency of atmospheric neutral and ion escape dynamics on short and long timescales upon solar wind and radiation variabilities.

In the following, we briefly introduce the science context and the scientific objectives of MEMO. The reader is referred to [21] for detailed scientific objectives. These objectives directly constrain the measurement requirements, that will be fulfilled by the MEMO instrumentation and appropriate orbital design.

\section{Science case}

The Martian atmosphere is mainly dominated by three cycles, water, carbon dioxide and dust, These cycles are characterised by exchanges between various 
atmospheric and surface reservoirs. These cycles are associated with expected isotopic and compositional signatures that must be characterized to reconstruct past climates. To understand processes at work now and in the past, they must be observed and modeled in details. Although current General Circulation Model (GCM) simulations satisfactorily reproduce the water seasonal climatology [25], there are numerous questions which can not been solved without new measurements. MEMO achieves a 3D mapping of the water cycle. This is combined with $3 \mathrm{D}$ measurements of the temperature, atmospheric transport, and oxidizing chemical species. Possible diurnal variations in the water column abundance and vertical profile are investigated. For the first time, the deuterium cycle is monitored. Condensation processes (which are related to the solar activity) induce isotopic fractionation, with strong temporal, vertical and meridional gradients in the $\mathrm{HDO} / \mathrm{H}_{2} \mathrm{O}$ ratio [26]. This ratio at the exobase level also constrains the ratio of the escape rate of $\mathrm{D}$ to $\mathrm{H}$ atoms. Characterization of the differences in the $\mathrm{H}_{2} \mathrm{O}$ and HDO cycles will increase our ability to understand how this affects the $\mathrm{H}$ and $\mathrm{D}$ escape rates, and to consequently infer the amount of water that escaped the planet.

Another important aspect of the Martian atmosphere is the photochemical cycle, that is thought to produce species that have oxidized and sterilized the Martian surface. The photochemical cycle also controls the water abundance at the exobase, hence the planetary water escape. As a consequence the photochemical cycle is a key element to understand the evolution of the Martian atmosphere and surface. MEMO simultaneously monitors $\mathrm{H}_{2} \mathrm{O}_{2}$, $\mathrm{H}_{2} \mathrm{O}, \mathrm{CO}$ and $\mathrm{O}_{3}$. These measurements are combined to wind measurements, which help to characterize the mean transport fluxes in the atmosphere. The Martian global atmospheric circulation is governed by a dynamical regime similar to that of the Earth. At solstices, the Hadley cell presents an ascending branch in the summer hemisphere and a descending branch in the winter hemisphere. At equinoxes, two Hadley cells ascend from the equatorial region transporting heat towards both northern and southern mid-latitudes. The general circulation is however modulated by several atmospheric waves, among which are the thermal tide (diurnal and semi-diurnal period) controlling the dynamics above $50 \mathrm{~km}$ altitude, and the travelling planetary waves, affecting the dust and condensable species transport. The vertical extent of the different cells and waves is very important, reaching $120 \mathrm{~km}$. In addition to the wind measurements, MEMO extend the temperature measurements from 0-60 to $0-120 \mathrm{~km}$. Their joint analysis provide an unprecedented and complete view of the Martian atmospheric dynamics and of the atmosphere-thermosphereexosphere coupling processes.

The past magnetic field of Mars can be explored through its remanent signature frozen into the magnetic minerals of the lithosphere. The measured remanent crustal magnetic field is symptomatic of a strong magnetic dynamo that was active some time in the past. The complete low altitude characterization of this signature will allow the past active magnetic field to be fully described and its influence upon the surface conditions and planetary evolution to be understood. The present-day magnetic field of Mars has a lithospheric origin, 
and is one to two orders of magnitude larger than the lithospheric magnetic field of the Earth [19]. Magnetic minerals froze the properties (direction and strength) of the ambient magnetic field when cooling down below their Curie temperature, and did not loose this information unless shocked or reheated, by impacts and volcanoes for instance. Lithospheric fields are heterogeneously distributed over the surface of Mars. Their discovery is one of the outstanding discoveries brought by MGS NASA mission. To first order, the northern hemisphere lowlands are almost devoid of magnetic signals, while the cratered southern hemisphere highlands are associated with strong magnetic fields. The largest volcanic edifices, as well as the largest impact basins, are devoid of significant magnetic anomalies at satellite altitude, although some volcanoes are still magnetized [20, 22]. Magnetic sources are located within the first tens of kilometres of the lithosphere [29], possibly covered by superficial, possibly a few kilometres thick, non magnetic material. These strong lithospheric fields interact with the solar wind and the Martian atmosphere, generating highly variable external fields.

Current measurements and models have allowed the lithospheric magnetic field of Mars to be described down to wavelength of about $300 \mathrm{~km}$ [34]. The magnetic signature of the lithosphere is very likely due to an ancient earthlike dynamic magnetic field. It was active in the early stages of Mars, probably during the first $0.5 \mathrm{Gyr}$, between the accretion of Mars and the occurrence of the very large impacts. This scenario also highlights the importance of the global changes which may be related to the formation of the huge $4,000-\mathrm{km}$ wide volcanic complex Tharsis [28] or to the late heavy bombardment which both occurred at about the same epoch [14,31].

By comparing the magnetic signatures of large ( $>100 \mathrm{~km}$ in diameter) impact craters, it should be possible to estimate at what epoch the dynamic magnetic field ceased, removing the global shielding for the atmosphere. When occurring in a magnetized lithosphere, impacts will induce a demagnetization, by thermal and shock effects [16], but also by excavation of material within the crater [35]. A 100-km diameter transient crater will be associated with a 3-nT magnetic signal at 150-km altitude. Only a complete mapping of Mars at such altitudes, with improved measurement accuracy, will allow these weak signatures to be correctly identified.

Complementary to the magnetic field characterization, information can be gained from the study of the gravity field variations through their effects on the velocity of the spacecraft. Although high altitude measurements have provided a global view of the gravity field anomalies [18], lower altitude passes on designated targets such as volcanoes or other tectonic structures will be used to constrain the absolute thickness of the Martian elastic lithosphere. Information on deeper structures will be deduced by studying the electromagnetic answer of the planet to the time variable magnetic fields [27]. This analysis is very sensitive to data quality, especially in terms of holes and jumps in data series. Another important aspect is the knowledge of the morphology and time variability of the inducing signal, i.e. the magnetic field external of external origin which, in turn, requires a simultaneous monitoring of interplanetary magnetic field. 
Because there is no planetary-scale magnetic field, the solar wind directly interacts with the atmosphere, resulting in a large-scale draping magnetic field in the dayside and a two-lobed tail structure in the night-side. The magnetic pile-up boundary shows a large variability, depending on the local time and season [7] but also on the direction and pressure of the solar wind. Simultaneous observations of plasma and magnetic field are missing. Above $500-\mathrm{km}$ altitude, the exosphere is predominantly produced by the present nonthermal atmospheric escape processes, which are at the origin of $\mathrm{H}_{2} \mathrm{O}$ and $\mathrm{CO}_{2}$ escapes [9]. These escape mechanisms strongly depend on both thermosphere and exosphere compositions, temperatures and densities [11]. In addition, the escape processes at work create magnetic perturbations. In situ measurements of the escaping flux (composition, energy, and density) help to understand their variability with respect to the solar EUV and wind conditions.

The escape rates are highly variable and strongly depend on the external conditions, namely, solar EUV and solar wind flux. To correctly reconstruct this dependence is critical for extrapolation of the current escape measurements backwards to evaluate the planetological consequences of the nonthermal escape processes related to the solar wind interactions. Clearly, a combination of the escape measurements inside the induced magnetosphere with simultaneous monitoring of the solar and solar wind conditions greatly helps such a reconstruction. Moreover, the consequences of solar energetic particle events (SEP) at Mars is still debated [21, 23]. Recent measurements onboard Mars Express showed that during solar flares combined with SEP the escape rates may increase by a factor of 10 [13]. Therefore, simultaneous monitoring of the solar energetic particle flux and escape fluxes would provide key information to understand their present and past role on the atmospheric evolution.

Up to now, solar wind parameters have been approximated a posteriori from estimates of the interplanetary magnetic field direction, by looking at areas where the lithospheric magnetic field is thought to be very weak [33]. This is why it is necessary to simultaneously monitor the solar wind, preferably upstream, during the MEMO mission. These measurements are of course used to constrain the solar wind, but also to improve the quality of the measurements of the lithospheric magnetic field, by identifying the time periods during which the solar activity is low. This also improves the characterization of the external magnetic field, to better infer the conductivity structure of the planet.

\section{Measurement requirements}

The scientific objectives of the MEMO mission imply a very specific orbital parameters, so that the distribution of the measurements is optimized in terms of location, altitude, local time, and season. The main spacecraft is placed on a low periapsis orbit $(130 \times 1,000 \mathrm{~km})$, while the micro-satellite has a much higher apoapsis $(10,000 \mathrm{~km})$. 


\subsection{Orbit requirements}

The orbit of MEMO is designed with a maximum apoapsis altitude of $1,000 \mathrm{~km}$, in order to reach a good signal-to-noise ratio as well as an optimal spatial resolution for the remote sensing instruments. The periapsis altitude is set to $130 \mathrm{~km}$, making it possible for the in situ instruments to probe the whole thermosphere. These orbital parameters are also dictated by the required low-altitude mapping of the magnetic field of the lithosphere. The nominal mission duration is set to one Martian year. MEMO science goals related to atmospheric observations imply a complete coverage in local time and latitude at least times a year. During this initial period, orbit maintenance manoeuvres are performed. The main spacecraft will thereafter be placed on a higher periapsis orbit, for an extended mission. More details on the orbit requirements can be found in [21].

\subsection{Measured parameters and instruments}

Table 1 provides the list of scientific questions, the strategy to answer to them and the list of instruments and measurement requirements adapted to reach it. Table 2 provides the list of instruments that fulfil the requested measurements.

\subsection{Micro-satellite}

Upstream solar wind and EUV/UV flux parameters are the keys: to understand the Martian environment response against variable solar wind, in particularly, for extreme conditions, to determine solar wind E-field which organizes the ion escape flux global distribution and to determine the contribution of the solar wind B-field to the magnetic field measurements at low altitude. Therefore, in order to add value to MEMO observations, a micro-satellite for solar wind monitoring is foreseen in the design of the mission. The microsatellite orbit is a trade-off between increasing time spent outside the induced magnetosphere and minimizing the distance to MEMO for communication purposes. The chosen orbit $(300 \times 10,000 \mathrm{~km})$ provides $90 \%$ time outside the induced magnetosphere and sufficient communication link.

\section{Model payload}

4.1 Main platform

MEMO payload is composed of:

- An "exospheric-upper atmosphere package" including low and high energetic ion spectrometers, thermal and energetic neutral spectrometers, a UV airglow spectrograph and an X-ray mapping spectrometer. 
- A "magnetic field package", including a magnetometer and a plasma package consisting of an electron spectrometer completed by plasma wave detectors (Mutual Impedance and Dual Langmuir Probes and a Magnetic Search Coil).

- A "low-middle atmosphere package", including a wide-field micro-camera, an IR dust analyzer and a sub-millimeter spectrometer.

Below $150 \mathrm{~km}$, the electron, neutral and low energy ion spectrometers work with high resolution mode. The EUV-FUV spectrograph and X-ray Mapping Spectrometer do not operate below $150 \mathrm{~km}$. The energetic neutral analyzer (ENA) operate above $300 \mathrm{~km}$. All other instruments, namely, the sub-millimetric spectrometer, the infrared dust analyzed, the micro-camera, the high energy ion mass spectrometer, the neutral spectrometer, the Dual Langmuir probe, the Magnetic Search Coil, the Mutual Impedance Probe and the vector magnetometers operate during the whole orbit. Micro-satellite payload operates continuously.

\subsection{Exospheric-upper atmosphere package}

Neutral mass and energy spectrometer is composed of a large size ionization source, by an electrostatic analyser and a time of flight (TOF)/carbon foil imager. The STOP is provided by an imager detector which also provides the localization for the impact which is related to the energy of the incident particles. The TOF measurement provides the mass. A new technology based on nano-tubes for the ionization source will provide the needed high sensitivity to measure the exospheric density. This instrument will measure continuously and simultaneously the energy and mass distributions.

EUV-FUV Airglow Spectrometer is a double spectrometer based on the photon counting method and is realized using Micro Channel Plate (MCP) detectors with Resistive Anode Encoder (RAE). Typical photocathodes are CsI for the EUV range and CsTe for the FUV range. In order to prevent sensitivity losses which are critical in UV ranges, a minimum of reflexion is guaranteed inside the instrument using only an off-axis parabola and a set of holographic gratings. The main advantages of the MCP + RAE detectors are their very high sensitivity mainly due to a very low dark current. Photon counting is then easily achievable at typical experiment temperature $\left(-20^{\circ} \mathrm{C} / 40^{\circ} \mathrm{C}\right)$. Seven orders of magnitude for the detection are then a typical value and offer the monitoring of a wide range of emission. Another advantage is the optimization of the optical layout which allows on one hand a compact and relative light instrument and on another hand a very versatile instrument due to a rotating scan mirror at the entrance. This scanning mirror is also very helpful to maintain the line-of-sight close to the limb during long integrations, to vertically scan the atmosphere independently on $\mathrm{S} / \mathrm{C}$ orbit and to extend the scanning vertical range. It allows also on flight star calibration. Due to the very high sensitivity of the MCP + RAE detectors, the instrument is only devoted to the very faint emissions. A big effort must be then taken to avoid direct illumination of the detectors 


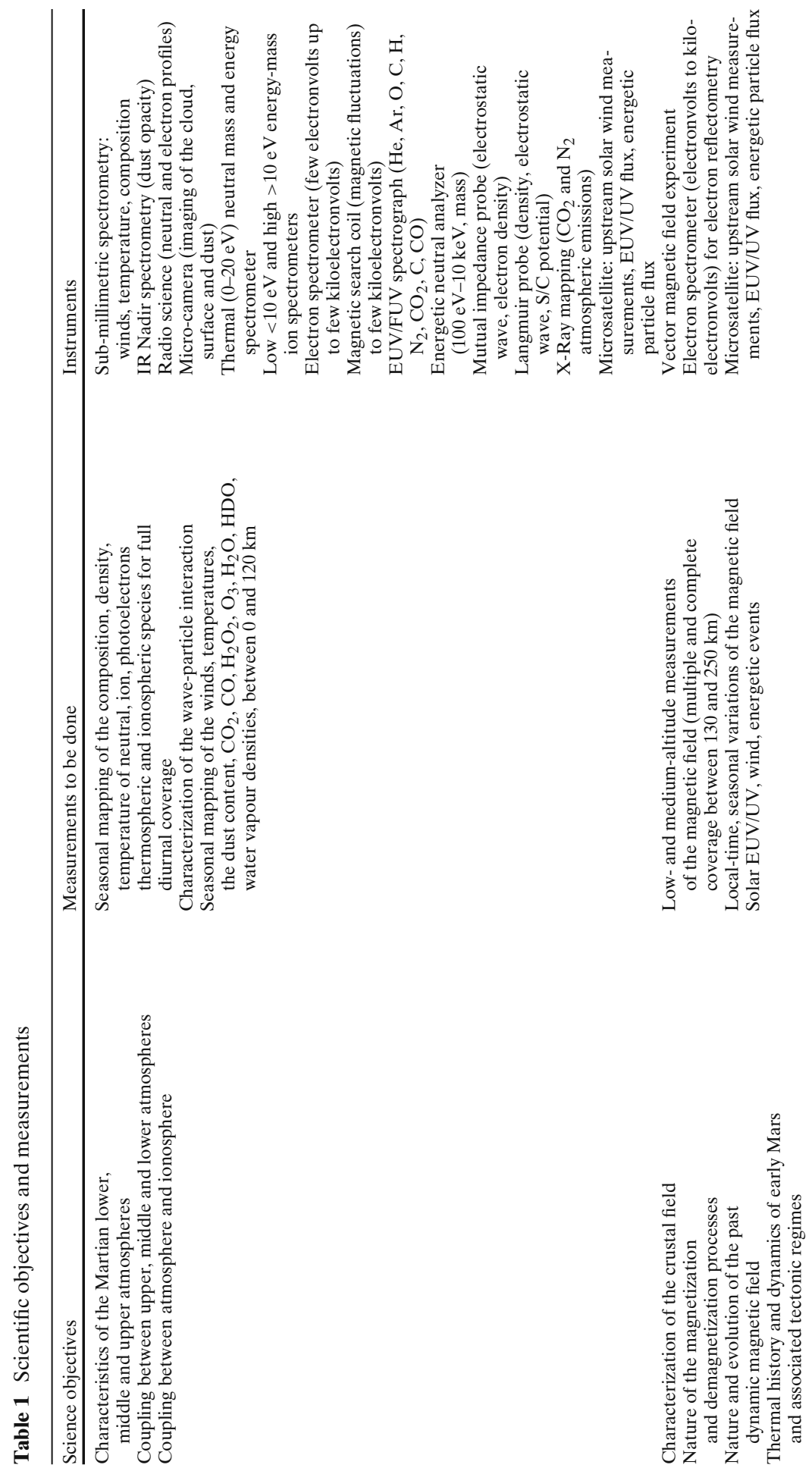



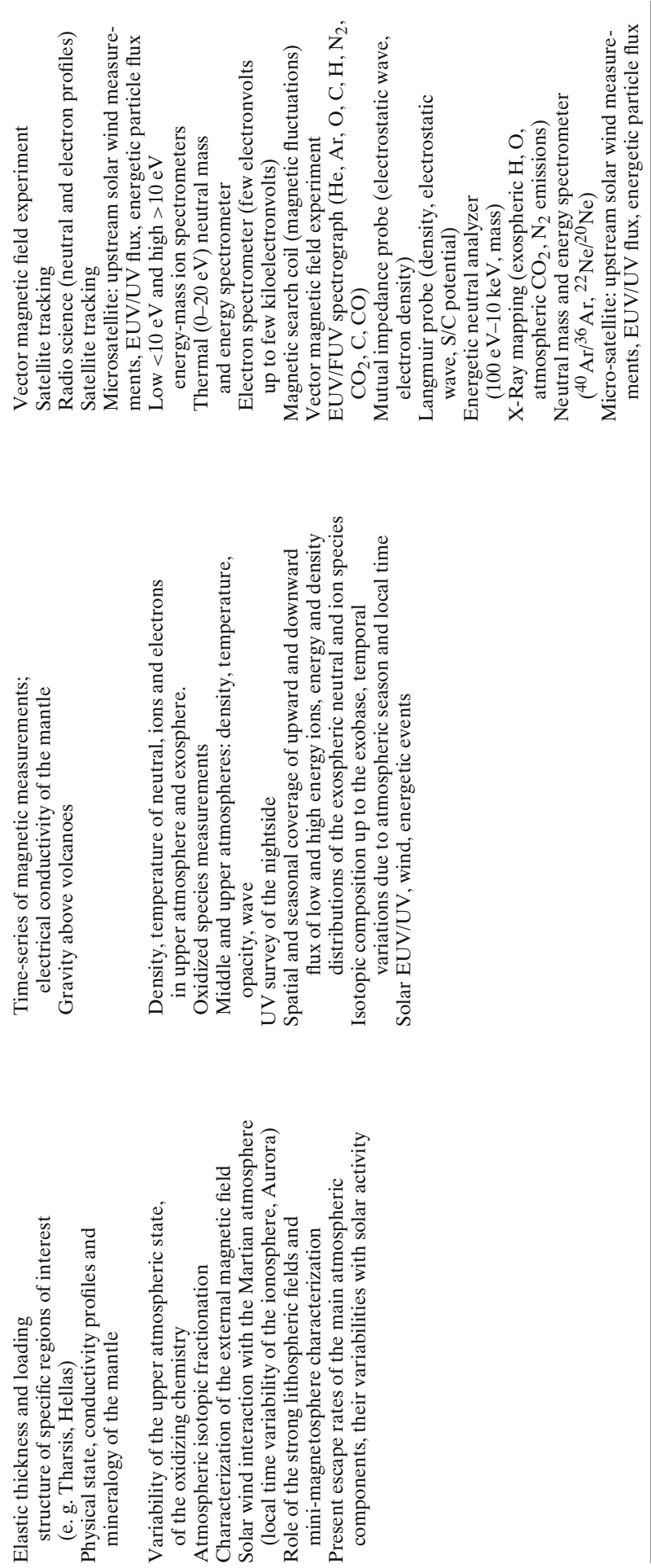


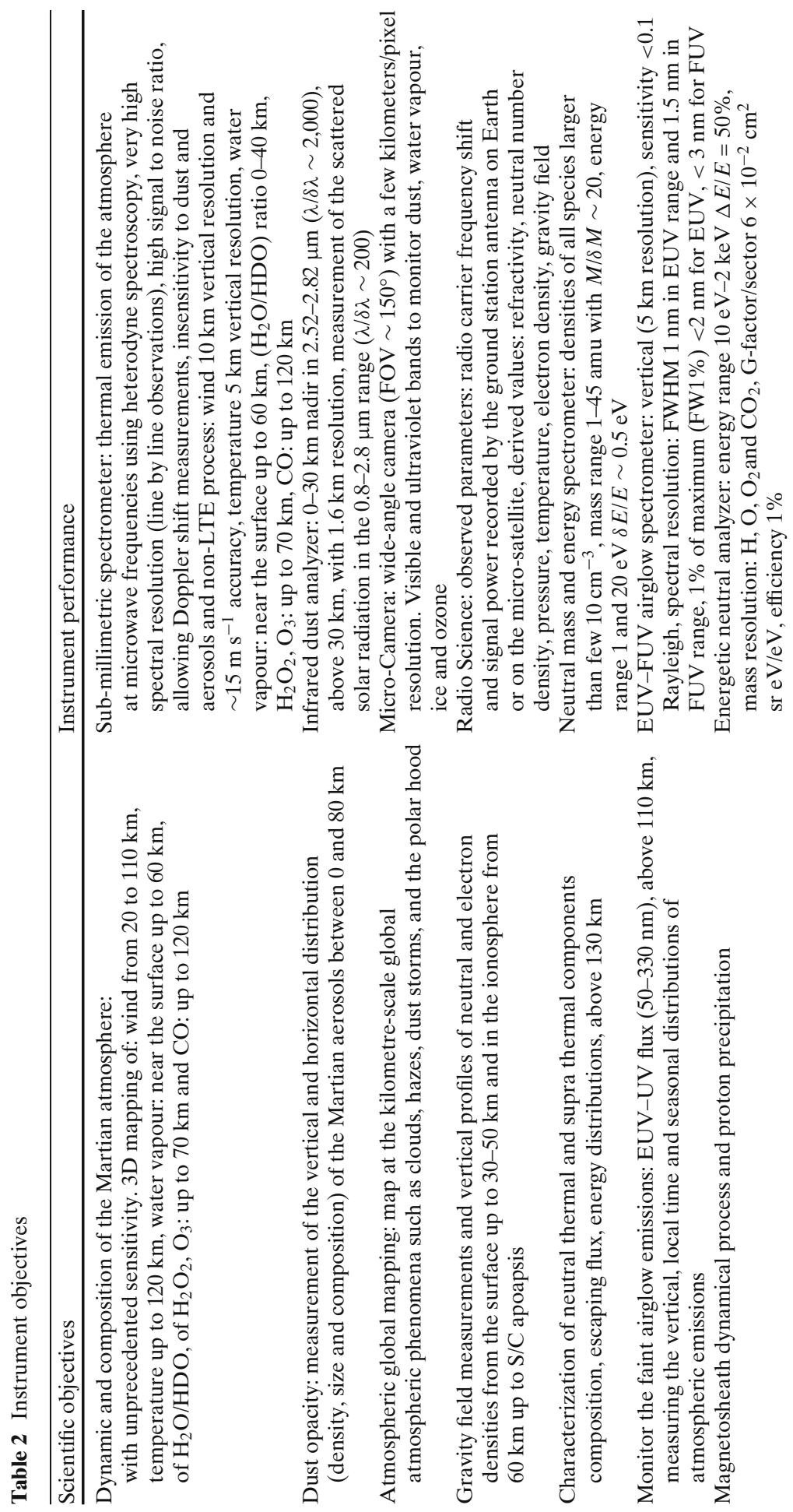



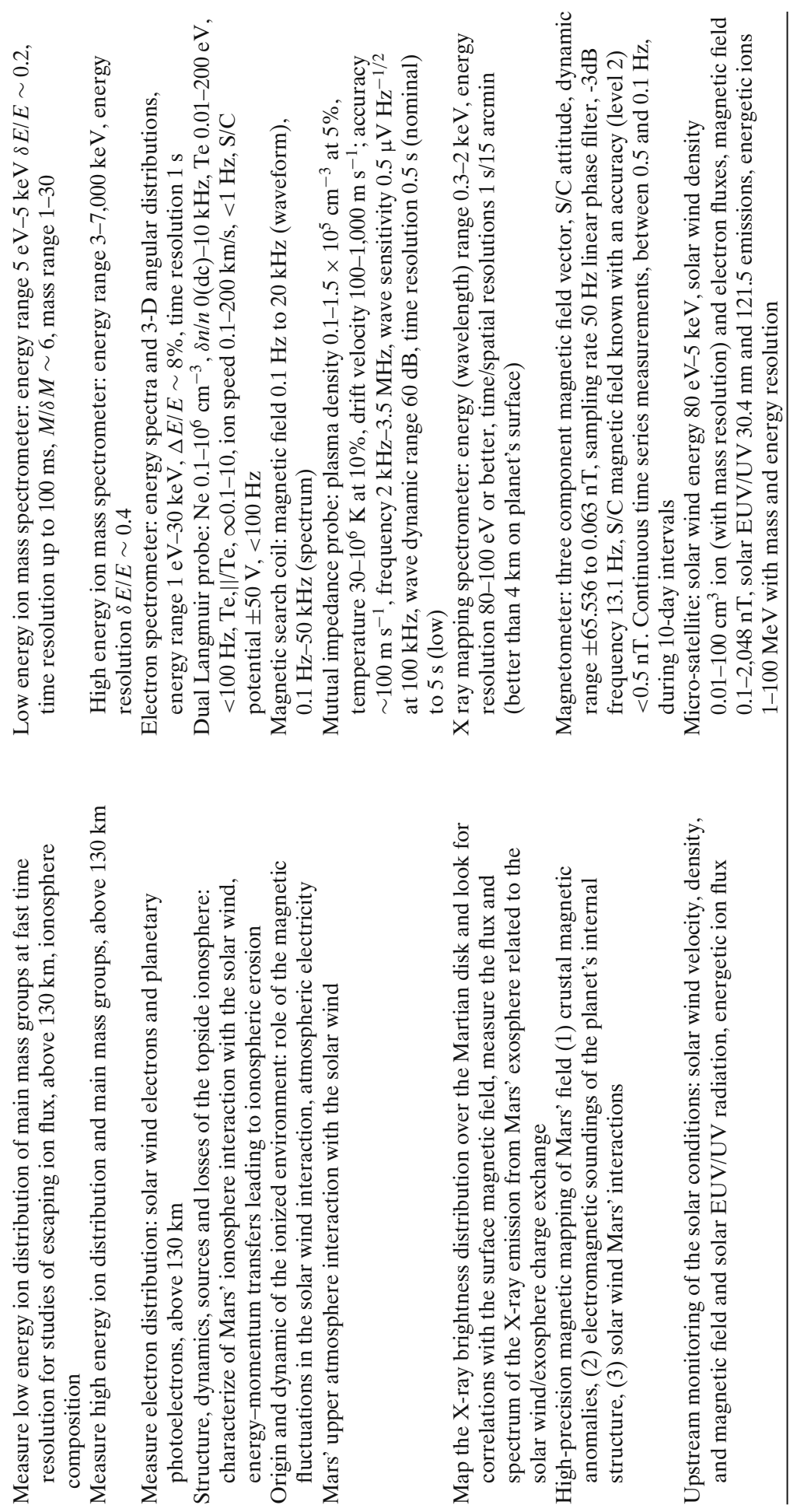
by strong light sources. This effort induces at instrument level the need of several baffles.

$X$ ray Mapping Spectrometer is composed of focusing micro-pore X-ray optics in conjunction with a digital imaging X-ray detector. So far planetary X-ray measurements have only been carried out remotely (i.e. from Earthorbiting X-ray observatories). In-situ measurements will provide higher spatial resolution, higher statistical quality spectra, with improved energy resolution. It will be no longer snapshots, but prolonged measurements which will allow us to map the X-ray emission from the Martian disk. In order to make measurements of the X-ray emission from the exosphere, selected pointing outside the Martian disk will be needed. To calibrate the energy response, an on board calibration source is foreseen. The field uniformity over time will need to regularly point the instrument on a known cosmic source.

Energetic Neutral Analyzer consists of four subsystems: an ion rejection system, an ionization surface, a photon rejection system which also performs crude energy analysis, and a velocity analysis section [17]. Neutrals enter the sensor through an electrostatic-charged particle deflector, which rejects ambient charged particles by a static electric field. The incoming neutrals are converted to positive (or negative) ions on an ionization surface and then pass through an electrostatic analyzer of a specific ("wave") shape that effectively blocks photons. The electrostatic analyzer also provides crude energy analysis. The "wave" analyzer design is similar to that used in the MTOF sensor of

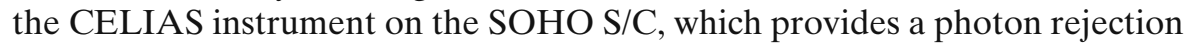
factor of $2 \times 10^{-8}$. Since the instrument must be capable of measuring masses up to 44 no foil can be used in the following TOF section. To measure the particle velocity (mass) the surface reflection principle is used. After exiting the electrostatic analyzer, neutrals are post-accelerated up to energy of $1.5 \mathrm{keV}$ and impact on a START surface under a grazing angle of $15^{\circ}$. During the impact, kinetic secondary electrons are emitted and the particles are reflected towards the STOP MCPs. The START and STOP timing give the particle velocity. Combining TOF measurements and the "wave" analyzer settings one determines the energy and mass.

High Energy Ion mass spectrometer is composed of two arrays of silicon semiconductor detectors (SSDs), each $0.1 \mathrm{~cm}^{2}$ area and $500 \mu \mathrm{m}$ thick, that measures the energy spectrum and angular distribution of pickup ions over more than half of the Sunward hemisphere. The first array of eight SSDs measures the energy spectrum and angular distribution of pickup ions in a $160 \times 20^{\circ}$ field of view that scans the sun-facing hemisphere with $20 \times 20^{\circ}$ resolution once per orbit. The second array of four SSDs covers an $80 \times 80^{\circ}$ field of view, with $20^{\circ}$ resolution in a direction orthogonal to the first array. This second array is used to resolve ambiguities between time and solar zenith angle during the half orbit needed to scan the FOV across the sun-facing hemisphere. Strips of four SSDs in a row on a single Si wafer, as developed for the STEREO IMPACT experiment, are used for MEMO, mounted behind $0.25 \mathrm{~cm}^{2}$ apertures to define the pinhole fields of view (FOVs). Rad-hard, 
low power $(5 \mu \mathrm{W}$ per SSD) application-specific integrated circuits (ASIC) preamp/shaping amp/ADC electronics, matched to the passively cooled SSD's low leakage current and capacitance, provide an electronic threshold of $1.2 \mathrm{keV}$. With the entrance windows of $<50 \mathrm{~nm} \mathrm{Si} \mathrm{equivalent,} \mathrm{incoming} \mathrm{H}, \mathrm{He}$, and $\mathrm{O}$ can be detected above 3,5 , and $8 \mathrm{keV}$, respectively, covering the entire pickup ion energy range, and up to $7 \mathrm{MeV}$. The counts are read out every $30 \mathrm{~s}$. Baffles and an occulting disk minimize sunlight contamination. Coincidence techniques reject the signals of penetrating particles from cosmic rays, and $\sim 300$-Gauss broom magnets (with yokes to suppress leakage field) at the entrance apertures deflect electrons away from the SSD's. In-flight calibration is provided by a ramp pulser.

Low Energy Ion Spectrometer consists of a number of Thomson parabola (E\| B analysers) located in a unit on the nadir facing side of the $\mathrm{S} / \mathrm{C}$. The advantage of this technique is simultaneous angle, energy and mass measurements over a $2 \pi$ field of view. The energy range of the sensor can be specified. The instruments must have a field-of-view free of any part of the S/C (including solar panels).

\subsection{Magnetic field package}

Magnetometer is composed of two tri-axial fluxgate vector magnetic field sensors (VFS) and two non-magnetic star tracker camera head units (CHU) co-mounted (VFS1 on the CHU baffle and VFS2 onboard) on a non-magnetic boom. The integrated miniature common data handling unit (DPU) is placed in the host $\mathrm{S} / \mathrm{C}$ and connected to the sensors via harness cables. The combination of two non-magnetic CHU and the primary vector field sensor (VFS), co-mounted on a temperature stable carbon fibre structure, offers a direct determination of the magnetic sensors' attitudes by stable mechanical transfer of the camera stellar orientation. The transfer is pre-flight determined and inorbit verified over time. This configuration maintains simplicity of design and use, and features the high accuracy attitude information needed for magnetic field mapping, while it avoids the difficulties associated with attitude transfer along the boom from the $\mathrm{S} / \mathrm{C}$, caused by limited stiffness, thermal flexing, mechanical creep, etc. The second VFS is placed inboard on the boom for gradiometric estimate of an $\mathrm{S} / \mathrm{C}$ magnetic dipole moment and to correct for a potentially significant $\mathrm{S} / \mathrm{C}$ field perturbation in the science data. The inboard sensor is in-orbit calibrated against the outboard sensor and also provides redundancy, even if at a slightly reduced field and attitude accuracy level. The VFS cores use a stress-annealed amorphous soft-magnetic alloy ribbon specially developed for the Ørsted mission. Sampling the extreme symmetry of the magnetic saturation levels of the amorphous material allows determination of the magnetic zero levels in the three orthogonal sensors with extreme accuracy and low noise. The sensor nulling feedback currents are precise measures of the components of the external magnetic vector field. 
The star camera system records the constellations within the FOV, corrects for image deficiencies, compares the positions to a HIPPARCOS-derived catalogue, corrects for relativistic aberration and other errors, and delivers attitude quaternions based on least squares fits to all the stars in the FOV. It autonomously solves for "Lost-in-Space" at wake-up and SEU. Magnetometer sensors and camera head units should be mounted on a on a light-weight, low stiffness and near-critically damped radial deployment boom. Typical boom mass is of the order of $250 \mathrm{~g} / \mathrm{m}$ to $1 \mathrm{~kg} / \mathrm{m}$, including foaming material. Final boom technology, accommodation, and deployment strategies will have to be studied in conjunction with the magnetic cleanliness programme.

Magnetic Search Coil is composed of one sensor (MSC) and one separated preamplifier unit (MPA). The sensor MSC is made of a fine wire coil, typically 50,000 turns, wounded on a high permeability Fe-Ni material. The coil is a resonant circuit whose response is flattened by a secondary winding procuring a field feedback. The feedback is generated by the preamplifier MPA. A change in the AC magnetic field will induce a current in the wire which will be increased in MPA and processed by an electronic card. The response of the system is flat from few Hertz to several kilohertz. The bandwidth is slightly adjustable. The sensitivity is limited by the thermal noise of the sensor and by the electronic noise of the preamplifier input stage made of low noise matched Field Effect Transistors. The system is optimized between 10 and 2,000 Hz. An internal intelligence allows to use sophisticated procedures in order to select interesting electro-magnetic events, to count, to catalogue and to measure in details these events and to trigger other instruments to analyze the interdependence of the events detected by the different instruments.

Mutual Impedance Probe measures the electrical coupling of the transmitting antenna (composed of two transmitting electrodes) and the receiving antenna (composed of two receiving electrodes) through surrounding plasmas and identifies the electron density, temperature, and drift velocity from the features of the frequency response. In the passive mode, the MIDST has finally the capability of a plasma wave analyzer, between $2 \mathrm{kHz}$ and $3.5 \mathrm{MHz}$. The MIDST is mainly composed of an electronics board and a mutual impedance probe: a light comb sensor $(250 \mathrm{~g})$ which consists of receiving and transmitting electrodes supported by a carbon fibre bar, $80-100 \mathrm{~cm}$ in length. The mutual impedance probe performance, unlike that of other plasma diagnostic techniques is neither affected by the shape, cleanliness, finish and photoemissive properties of the sensor, nor by the lack of uniformity of its surface potential. Extremely low energetic plasmas can then be explored. The distance between the transmitting pole and the most distant receiving electrode must be at least of the order of $2 \mathrm{D}$ lengths. The Debye length $L_{\mathrm{D}}$, a characteristic length of the plasma, is proportional to $(\mathrm{Te} / \mathrm{Ne})^{1 / 2}$, where $\mathrm{Te}$ and $\mathrm{Ne}$ are the temperature and density of the thermal electron population. The interference structure and resonance peak that are used for plasma diagnostics are more pronounced for larger separation distances (or shorter $L_{\mathrm{D}}$ ), the only limitation in the accuracy then comes from the frequency resolution of the analyzer. 
Electron spectrometer consists of a pair of top hat electrostatic analysers located at two corners of the S/C. Electrostatic scanning will be used on each sensor $\left( \pm 45^{\circ}\right)$. Deployment of two such sensors allows the instrument full $4 \pi$ angular coverage. Alternatively, a single sensor solution could be used probably on a short boom, with the undeflected field of view to include the nadir direction. If accommodated on the spacecraft, the photo-electrons emitted by the spacecraft when illuminated by the solar flux would limit the capability of this instrument to measure low energy electrons.

Dual Langmuir Probe (DLP) consists of two $0.5-2 \mathrm{~m}$ solid deployable booms, with a few $\mathrm{cm}$ diameter TiN coated spherical sensors at the tips of the booms. A Langmuir probe gives primarily a measurement of the density of charged particles and a number of other derivable cold plasma quantities. Basically, a bias voltage is applied to a spherical sensor and the resulting current, which is proportional to the plasma charge density, is measured. To enable simultaneous measurements of electrons and ions, dual probes are used with one probe biased at a positive potential and the other at a negative potential. A dual probe system, when used with two sensors on equal potentials, also makes it possible to perform interferometry measurements. By varying the probe potential, the electron and ion temperatures, $\mathrm{S} / \mathrm{C}$ potential, average ion mass, and ion ram speed, can be derived. Normally, this is performed by a socalled Langmuir probe sweep, where the probe bias voltage is varied rather slowly, to yield a probe voltage-current characteristic or Langmuir curve, from which the aforementioned quantities are derived. The Dual Langmuir Probes employ a method that radically improves the time resolution compared to sweep measurements. By adding a small harmonic variation to the probe potential, it is possible to extract the instantaneous measurements of the first and second order derivatives of the voltage-current characteristic at constant probe bias voltage. From the first order derivative, instantaneous values of the derivable quantities are obtained. The second order derivative is used as a quality measure of the instantaneous observations. The harmonic variation method is also used in a feedback loop for detailed tracking of the zero current, which is used to determine the S/C potential to very high accuracy.

\subsection{Low-middle atmospheric package}

Sub-millimetric spectrometer analyzes the thermal emission of the atmosphere at microwave frequencies using heterodyne spectroscopy. In practice, such an instrument will perform measurements at the atmospheric limb and at nadir using a receiver dedicated to the monitoring of selected lines of key molecule around $320-350 \mathrm{GHz}$ (Table 2). Its main advantages are a very high spectral resolution (line by line observations), a high signal to noise ratio, allowing Doppler shift measurements, insensitivity to dust, aerosols, and Non-LTE process (unlike infrared). The instrument consists of coupled heterodyne receivers at sub-millimeter wavelength. It is designed for both continuum and spectroscopic observations. The foreseen telescope is a $23-\mathrm{cm}$ 
Gregorian antenna composed of a parabolic main reflector followed by a $45^{\circ}$-tilted flat sub-reflector. The antenna is placed inside a protective shroud structure and rotates around an axis which is along-track oriented. The observation is therefore a cross-track field of view scanning. The whole instrument is mounted rigidly on the orbiter $\mathrm{S} / \mathrm{C}$. The two heterodyne receivers should be based on uncooled $330 \mathrm{GHz}$ subharmonically pumped mixers using planar diode technology. A phase-locked ultra-stable oscillator is used to provide very high frequency stability for the spectroscopic measurements. A set of autocorrelator spectrometers (ACS) must be provided to observe large pressurebroadened spectral lines with 1-8 MHz resolution. A set of multichannel chirp transform spectrometer (CTS) with $100 \mathrm{kHz}$ resolution must be also provided to observe atmospheric spectral lines.

Infrared dust analyzer probes the solar light scattered in the near infrared at the limb by aerosols, and measure surface pressure in nadir viewing. The scientific investigations require two different channels: a limb channel with spectroscopic resolving power up to 200 in the $1.5-3 \mu \mathrm{m}$ spectral range with a moderate angular resolution (1 mrad) in a field of view of about $100 \mathrm{mrad}$, and a nadir channel with spectral resolution up to 2,000 to map the $\mathrm{CO}_{2}$ surface pressure. Short exposure times are needed because of the S/C velocity as well as $\mathrm{S} / \mathrm{N}$ ratios of several hundreds. Allowing for the elliptical orbit of the $\mathrm{S} / \mathrm{C}$, the limb viewing channel must be mounted on a scanning platform.

Micro-Camera is a low resolution camera working on an extended spectral range, $250-1,000 \mathrm{~nm}$, utilizing a new detector technology based on CMOS. It may work in the UV and visible spectral range maintaining good quantum efficiency, $20 \%$ at $250 \mathrm{~nm}$ and $80 \%$ at $700 \mathrm{~nm}$, a fill factor of $100 \%$ and a full well of $10^{5}$ electrons. This new solution will allow to use a less fast optics reducing complication, as it has been adopted in the MARCI camera on board the Mars Climate Orbiter. It doesn't need to apply different coatings on different sections of the chip. The camera will be composed by two optical paths converging in the same FPA, in order to use lenses specific for UV and visible spectral range.

Radio Science computes the vertical refractivity profile from the surface up to a given altitude from the observed radio carrier frequency shift due to the bending and the occultation geometry. It is proposed a one-way radio link. The $\mathrm{S} / \mathrm{C}$ is transmitting the radio signal to Earth so that a highly stable frequency reference source is required in form of an Ultra Stable Oscillator (USO) which is externally connected to the transponders. The availability of a ground station on Earth at the time of experiment performance is required. Currently, all ESA and NASA DSN ground station equipments support such an experiment. The main advantages are a highly precise derivation of the neutral and electron density profiles at $\sim 500 \mathrm{~m}$ altitude resolution. This measurement is only feasible during Earth occultation seasons when the $\mathrm{S} / \mathrm{C}$ is occulted by the planetary disk in each orbit. It is also foreseen to realize radio science occultation between the micro-satellite and MEMO main platform to provide electron density profiling on the nigh side, a region not-achievable for the ground based radio science. 


\subsection{Micro-satellite}

The MEMO micro-satellite is a detached and autonomously flying solar monitoring instrument. It communicates with MEMO via dedicated link and from the main satellite interface it looks like an ordinary payload. Microsatellite is a spin-stabilized with a spin rate around $1 \mathrm{rpm}$ providing sufficient gyroscopic stiffness to resist disturbance torques and fulfil payload requirements. The total mass of the micro-satellite is $25.3 \mathrm{~kg}$ including $1.8 \mathrm{~kg}$ of scientific instruments, and $5.6 \mathrm{~kg}$ for separation and communication systems on the main spacecraft.

The S/C structure consists of aluminium honeycomb panels with CFRP facings. It is an octagon shaped disk where the side panels are internal mounting area for units. This enables easy balancing of the units regarding the symmetry about the S/C spin axis and a modular design for other type of missions. The attitude determination system is based on a two-axis sun sensor, three-axis rate sensor-both engineered with MEMS (Micro-ElectroMechanical System) technology and a miniaturized horizon crossing indicator providing attitude accuracies $<1.5^{\circ}$. Attitude control is performed via a MEMS-engineered cold gas micro-propulsion system-dry mass $2 \mathrm{~kg}$ enabling theoretical manoeuvre accuracy of the order of $10^{-3}$ degrees. Total propellant mass is about $40 \mathrm{~g}$ for the nominal mission duration. The Electra Lite UHF transceiver, implemented on both spacecraft, secures orbit determination from the MEMO via two-way Doppler ranging and constantly send an ephemeris update to the micro-satellite. The end-of-life solar arrays generate around $130 \mathrm{~W}$ (20\% margin) at Mars aphelion. The overall system design of the micro-satellite is done in such way that the life-time can be much longer than nominal and the solar wind monitoring for other missions is an option as well.

Solar UV photometer provides the absolute flux from full solar disk measurements in He II $30.4 \mathrm{~nm}$ and $\mathrm{H} 121.6 \mathrm{~nm}$. It is aligned on the micro-satellite spin axis. The He II channel design is similar to SOHO/SEM instrument and uses a transmission grating and a silicon photodiode as a detector. The Lyman$\alpha$ channel is a standard photometer based on $\mathrm{MgF}_{2}$ windows, $\mathrm{D} / \mathrm{H}$ cell, and a channel electron multiplayer as a detector.

Plasma analyzer, an ion mass analyzer, is a compact electrostatic analyzer followed by a TOF cell for velocity (mass) identification. The TOF cell uses surface interactions to generate START and STOP signals. Its boresight is also aligned on the micro-satellite spin axis.

Radiation environment monitor is a $\Delta \mathrm{E}-\mathrm{E}$ telescope to measure flux, energy, and mass ( $\mathrm{H}, \mathrm{He}$, heavy) of energetic ions in the range 1-100 MeV. REM consists of three silicon detectors D1 ( $\triangle$ E-detector), D2 (E-detector), and D3 (back detector). The detector thickness and the front window are chosen in such a way that the valid event is given by D1 and D2 only. The events D1 and D2 and D3 are disregarded. The pulse height analysis of the signals from D1 and D2 gives particle energy and mass. The instrument boresight is co-aligned with the Parker spiral angle $57^{\circ}$ at Mars. 
Magnetometer (MAG) is a two fluxgate sensors mounted on $60 \mathrm{~cm}$ booms which are perpendicular to the spin axis. Two sensors are used for reliability and dynamical stability of the micro-satellite.

\subsection{Main platform magnetic cleanliness}

The magnetic sensor deployment distance (i.e. the length of the magnetometer boom) is determined by the science requirements in conjunction with the obtainable level of S/C magnetic field at the boom tip, quantified by a S/C centred equivalent magnetic dipole moment. If deployed into the dipolar field regime (about two to three $\mathrm{S} / \mathrm{C}$ diameters), results from previous experiments show that the dual-sensor gradiometry can compensate for $\sim 90 \%$ of the remaining S/C field at the boom tip. Scientific objectives in terms of lithospheric magnetization characterization require the Martian magnetic field to be resolved within an accuracy of $0.5 \mathrm{nT}$.

Three possible errors will contribute: intrinsic sensor error, attitude error, and S/C generated magnetic fields. The intrinsic sensor error associated with the VFS is $0.3 \mathrm{nT}$ in the long term, and lower than $0.1 \mathrm{nT}$ during one orbit. The attitude errors caused by attitude uncertainties can be easily estimated. Two CHUs are used, first to avoid loosing data because one CHU looks into the Sun or the planet. With only one CHU one may loose around $20 \%$ of the data. Associated error with two CHUs is of the order of a few arc seconds, resulting in an error $<0.03 \mathrm{nT}$ in a 1,000 nT field. Such a low error leaves an error of up to $0.49 \mathrm{nT}\left(0.5=\sqrt{\left(0.1^{2}+0.03^{2}+0.49^{2}\right)}\right.$ for the S/C generated magnetic fields at the end of the boom (after the $90 \%$ error reduction due the dual-magnetometer approach). Standard engineering methods, such as appropriate back-wiring layouts, the avoidance of softly magnetized material, and eventually magnetic shielding, will be used to minimize the equivalent magnetic moment of the S/C. We currently foresee a maximum boom length of $6 \mathrm{~m}$.

\section{Conclusions}

The present Martian atmosphere is either the residual of the atmosphere which formed at that time or is the residual of a period of sporadic outgassing which occurred few tens of million years ago or is constrained by a permanent equilibrium between present outgassing and loss to space. Theoretical works predict that ion and neutral escape rates are strongly dependent on the solar EUV intensity and solar forcing [9]. The past EUV flux is thought to have been up to 10 times larger 4.1 Gyr ago [30], whereas the solar wind flux may have been up to 100 times larger at the same epoch [36]. In these conditions, Mars should have lost several tens meters of water over geologic time [8] because of the past solar conditions and because it was not shielded by a global magnetic field. An integrated Martian system, coupling current interior 
Fig. 1 Schematic (artist) view of the MEMO mission. Two spacecrafts are placed on two orbits (with two different elipticities), to study the environment (the blurred and cloudy $E$ ) and the magnetic field (the magnetized $M$ ) of the planet Mars

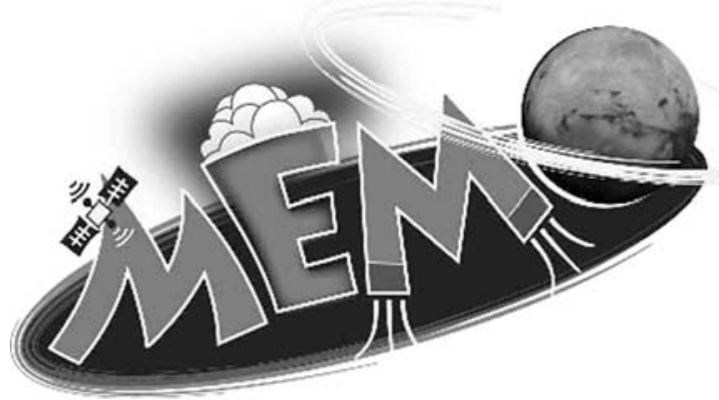

dynamics, past lithosphere evolution, surface volcanism and tectonics, climatic evolution, atmospheric escape, and solar conditions, is a major scientific objective to which the MEMO mission greatly contributes.

Several committees, including MEPAG and IMEWG, have recognized that determining the fate of water on Mars is a top priority in order to understand key questions concerning habitability and exobiology. The lost Japanese NOZOMI mission was originally developed to fill the gap in our understanding of planet-solar wind interaction. The recently pre-selected SCOUT missions will address the escape rate in the upper atmosphere but will provide neither a detailed picture of the main chemical, thermodynamical and dynamical cycles nor the necessary measurements of lithospheric magnetic anomalies. More generally, no past or presently scheduled exploration missions to Mars, such as the Mars Science Laboratory or Mars Reconnaissance Orbiter, plan to characterize the $70-120 \mathrm{~km}$ altitude region or to measure simultaneously the upper atmosphere, solar and magnetic field states.

MEMO (Fig. 1) is strongly supported by a large European scientific community and by Russian, Japanese and US scientists. A mission like MEMO ideally completes the present European programme for the Cosmic Vision and exploration of Mars by comprehensively contributing to our understanding of what has made the Earth suitable for life. Use of controlled aerobraking manoeuvres and comprehensive physical characterization of the lower atmosphere (0$100 \mathrm{~km}$ ) and space weather conditions at Mars including radiation dose are also of essential technical interest for the Exploration Programme. Unprecedented operation of a two-spacecraft constellation at Mars provides additional and necessary experience for the Exploration Programme.

Acknowledgements Michel Capderou (LMD, France) performed all the calculations needed to optimize MEMO orbit during the different scientific phases by providing us the global coverage of each potential orbit, the global propulsion budget needed to maintain them during one Martian year and also by providing us the global coverage of the radio-occultation operation between MEMO and the micro-satellite. The contributions of Emmanuel Hinglais and Regis Bertrand (CNES, France), and more generally the support from the CNES to the preparation of this project have been essential for the definition of the mission profile from the launch, up to the insertion and 
aerobraking phases of MEMO. MEMO logo was realized by Alain Cossard, LPGNantes, University of Nantes. Micro-satellite system design was performed at the Swedish Space Corporation (Solna, Sweden). Contribution from Nils Pokrupa (Swedish Space Corporation, Solna, Sweden) is also kindly acknowledged. At the end, we would like to thank Astrium and in particular Roger Foerstner (Astrium, Germany) to provide us details on the best strategy to reuse Mercury Planetary Orbiter/Bepi Colombo platform for the design of MEMO platform in order to minimize as much as possible the total cost of the project.

\section{References}

1. Acuña, M.H., Connerney, J.E.P., Wasilewski, P., Lin, R.P., Anderson, K.A., Carlson, C.W., McFadden, J., Curtis, D.W., Mitchell, D., Rème, H., Mazelle, C., Sauvaud, J.A., d'Uston, C., Cros, A., Médale, J.L., Bauer, S.J., Cloutier, P., Maihew, M., Winterhalter, D., Ness, N.F.: Magnetic field and plasma observations at mars: initial results of the mars global surveyor mission. Science 279, 1676-1680 (1998)

2. Acuña, M.H., Connerney, J.E.P., Ness, N.F., Lin, R.P., Mitchell, D., Carlson, C.W., McFadden, J., Anderson, K.A., Reme, H., Mazelle, C., Vignes, D., Wasilewski, P., Cloutier, P.: Global distribution of crustal magnetization discovered by the Mars Global Surveyor MAG/ER Experiment. Science 284, 790-793 (1999)

3. Acuña, M.H., Connerney, J.E.P., Ness, N.F., Lin, R.P., Mitchell, D., Carlson, C.W., McFadden, J., Anderson, K.A., Rème, H., Mazelle, C., Vignes, D., Wasilewski, P., Cloutier, P.: The magnetic field of mars: summary of results from the aerobraking and mapping orbits. J. Geophys. Res. 106(E10), 23403-23418 (2001)

4. Barabash, S., Fedorov, A., Lundin, R., Sauvaud, J.-A.: Martian atmospheric erosion rates. Science 315, 501-503 (2007)

5. Bibring, J.-P., Squyres, S.W., Arvidson, R.E.: Merging views on Mars. Science 313, 1899-1901 (2006)

6. Bibring, J.-P., Langevin, Y., Mustard, J.F., Poulet, F., Arvidson, R.E., Gendrin, A., Gondet, B., Mangold, N., Pinet, P., Forget, F.: OMEGA team: Global mineralogy and aqueous Mars history derived from OMEGA/Mars Express data. Science 312, 400-404 (2006)

7. Brain, D.A., Halekas, J.S., Lillis, R., Mitchell, D.L., Lin, R.P., Crider, D.H.: Variability of the altitude of the Martian sheath. Geophys. Res. Lett. 32, L18203.1-L18203.4 (2005). doi:10.1029/2005GL023126

8. Carr, M.H., Head, J.W.: Oceans on Mars: An assessment of the observational evidence and possible fate. J. Geophys. Res. 108 (2003). doi:10.1029/2002JE001963

9. Chassefière, E., Leblanc, F.: Mars atmospheric escape and evolution: interaction with the solar wind. Planet. Space Sci. 52, 1039-1058 (2004)

10. Chassefière, E., Leblanc, F., Langlais, B.: The combined effect of escape and magnetic field histories at Mars. Planet. Space Sci. 55, 343-357 (2007)

11. Chaufray, J.-Y., Modolo, R., Leblanc, F., Chanteur, G., Johnson, R.E., Luhmann, J.G.: Mars solar wind interaction: formation of the Martian corona and atmospheric loss to space. J. Geophys. Res. 112 (2007). doi:10.1029/2007JE002915

12. Connerney, J.E.P., Acuña, M.H., Wasilewski, P.J., Ness, N.F., Rème, H., Mazelle, C., Vignes, D., Lin, R.P., Mitchell, D.L., Cloutier, P.A.: Magnetic lineation in the ancient crust of Mars. Science 284, 794-798 (1999)

13. Futaana, Y., Barabash, S., Yamauchi, M., McKenna-Lawlor, S., Lundin, R., Luhmann, J.G., Brain, D., Carlsson, E., Sauvaud, J.-A., Winningham, J.D., Frahm, R.A., Wurz, P., Holmström, M., Gunell, H., Kallio, E., Baumjohann, W., Lammer, H., Sharber, J.R., Hsieh, K.C., Andersson, H., Grigoriev, A., Brinkfeldt, K., Nilsson, H., Asamura, K., Zhang, T.L., Coates, A.J., Linder, D.R., Kataria, D.O., Curtis, C.C., Sandel, B.R., Fedorov, A., Mazelle, C., Thocaven, J.-J., Grande, M., Koskinen, H.E.J., Sales, T., Schmidt, W., Riihela, P., Kozyra, J., Krupp, N., Woch, J., Fränz, M., Dubinin, E., Orsini, S., Cerulli-Irelli, R., Mura, A., Milillo, A., Maggi, M., Roelof, E., Brandt, p., Szego, K., Scherrer, J., Bochsler, P.: Mars express and Venus express multi-point observations of geoeffective solar flare events in December 2006. Planet. Space Sci. 56, 873-880 (2008) 
14. Gomes, R., Levison, H., Tsiganis, K., Morbidelli, A.: Origin of the cataclysmic late heavy bombardment of the terrestrial planets. Nature 435, 466-469 (2005)

15. Hartmann, W.K., Neukum, G.: Cratering chronology and the evolution of Mars. Space Sci. Rev. 96, 165-194 (2001)

16. Hood, L.L., Richmond, N.C., Pierazzo, E., Rochette, P.: Distribution of crustal magnetic fields on Mars: shock effects of basin-forming impacts. Geophys. Res. Lett. 30, 14.1-14.4 (2003). doi:10.1029/2002GL016657

17. Kazama, Y., Barabash, S., Wieser, M., Asamura, K., Wurz, P.: Development of a LENA instrument for planetary missions by numerical simulations. Planet. Space Sci. 55, 1518-1529 (2007)

18. Konopliv, A.S., Yoder, C.F., Standish, E.M., Yuan, D.-N., Sjogren, W.L.: A global solution for the Mars static and seasonal gravity, Mars orientation, Phobos and Deimos masses, and Mars ephemeris. Icarus 182, 23 (2006)

19. Langlais, B., Purucker, M.E., Mandea, M.: The crustal magnetic field of Mars. J. Geophys. Res. 109, E02008.1-E02008.16 (2004). doi:10.1029/2003JE002058

20. Langlais, B., Purucker, M.E.: A polar magnetic paleopole associated with Apollinaris Patera, Mars. Planet. Space Sci. 55, 270-279 (2007)

21. Leblanc, F., Langlais, B., Fouchet, T., Barabash, S., Breuer, D., Chassefière, E., Coates, A., Dehant, V., Forget, F., Lammer, H., Lewis, S., Lopez-Valverde, M., Mandea, M., Menvielle, M., Pais, A., Paetzold, M., Read, P., Sotin, C., Tarits, P., Vennerstrom, S.: Mars Environment and Magnetic Orbiter: science and measurements objectives. Astrobiology (2008, in press)

22. Lillis, R.J., Manga, M., Mitchell, D.L., Lin, R.P., Acuña, M.H.: Unusual magnetic signature of the Hadriaca Patera volcano: implications for early Mars. Geophys. Res. Lett. 33, L03202.1L03202.4 (2006). doi:10.1029/2005GL024905

23. Luhmann, J.G., Kasprzak, W.T., Russel, C.T.: Space weather at Venus and its potential consequences for atmosphere evolution. J. Geophys. Res. 12(E4), E04S10 (2007). doi:10.1029/2006JE002820

24. Milliken, R.E., Mustard, J.F., Poulet, F., Jouglet, D., Bibring, J.-P., Gondet, B., Langevin, Y.: Hydration state of the Martian surface as seen by Mars express OMEGA: 2-H2O content of the surface. J. Geophys. Res. 112 (2007). doi:10.1029/2006JE2853

25. Montmessin, F., Forget, F., Rannou, P., Cabane, M., Haberle, R.M.: Origin and role of water ice clouds in the Martian water cycle as inferred from a general circulation model. J. Geophys. Res. 109, E10004.1-E10004.26 (2004). doi:10.1029/2004JE002284

26. Montmessin, F., Fouchet, T., Forget, F.: Modeling the annual cycle of HDO in the Martian atmosphere. J. Geophys. Res. 110, E03006.1-E03006.16 (2005). doi:10.1029/2004JE002357

27. Olsen, N.: Induction studies with satellite data. Surv. Geophys. 20, 309-340 (1999)

28. Perron, J.T., Mitrovica, J.X., Manga, M., Matsuyama, I., Richards, M.A.: Evidence for an ancient Martian ocean in the topography of deformed shorelines. Nature 447, 840-843 (2007)

29. Quesnel, Y., Langlais, B., Sotin, C.: Local inversion of magnetic anomalies: implications for Mars' crustal evolution. Planet. Space Sci. 55, 258-269 (2007)

30. Ribas, I., Guinan, E.F., Güdel, M., Audard, M.: Evolution of the solar activity over time and effects on planetary atmospheres: I. High energy irradiances (1-1700 A). Astrophys. J. 622, 680-694 (2005)

31. Solomon, S.C., Aharonson, O., Aurnou, J.M., Banerdt, W.B., Carr, M.H., Dombard, A.J., Frey, H.V., Golombek, M.P., Hauck, S.A., Head, J.W., Jakosky, B.M., Johnson, C.L., McGovern, P.J., Neumann, G.A., Phillips, R.J., Smith, D.E., Zuber, M.T.: New perspective on ancient Mars. Science 307, 1214-1220 (2005)

32. Stevenson, D.J.: Mars' core and magnetism. Nature 412, 214-219 (2001)

33. Vennerstrom, S., Olsen, N., Purucker, M., Acuña, M.H., Cain, J.C.: The magnetic field in the pile-up region at Mars, and its variation with the solar wind. Geophys. Res. Lett. 30 (2003). doi:10.1029/2003GL016883

34. Whaler, K.A., Purucker, M.E.: A spatially continuous magnetization model for Mars. J. Geophys. Res. 110, E09001.1-E09001.11 (2005). doi:10.1029/2004JE002393

35. Wieczorek, M.A., Phillips, R.J.: Lunar multiring basins and the cratering process. Icarus 139, 245-259 (1999)

36. Wood, B.E., Müller, H.-R., Zank, G.P., Linsky, J.L., Redfield, S.: New mass-loss measurements from astrospheric Ly- $\alpha$ absorption. Astrophys. J. 628, L143 (2005) 